\title{
Multi-time scale optimization scheduling of integrated energy system considering demand side response
}

\author{
Peng Fang ${ }^{1}$, Cui Mao ${ }^{2, *}$, Yuping Chen², Shan Zhou², Rui You ${ }^{2}$, Danning Xu \\ ${ }^{1}$ Zhejiang Electric Power Industry Association, No. 8, Huanglong Road, Xihu District, Hangzhou City, Zhejiang Province \\ ${ }^{2}$ Zhejiang Huayun Electric Power Engineering Design consulting Co. LTD,No. 1, Huadian Lane, Xiacheng District, Hangzhou City, \\ Zhejiang Province
}

\begin{abstract}
As the physical carrier of the energy Internet, the integrated energy system has become the focus of current research. Considering the renewable energy and demand side load fluctuations, using the price type and the alternative demand side response characteristics, a day-ahead and intraday optimization scheduling model that takes into account the demand side response is established, in which the intraday, according to the difference of electricity, cold/heat and natural gas scheduling time, a three-layer rolling optimization scheduling model is proposed. The example analysis shows that this model can suppress the fluctuation of renewable energy and load in the day, improve the stability of the system, and further reduce the operating cost of the system.
\end{abstract}

\section{Introduction}

With the rapid development of economy, human beings are facing the problems of fossil energy depletion and environmental pollution. The regional integrated energy system (RIES) integrates and manages electric energy, natural gas, thermal energy and various new energy sources which can realize the cascade utilization of energy and improve the energy utilization efficiency. A large number of literatures have studied the optimal operation of RIES. Reference [1] introduced the penalty coefficient of wind curtailment and net load fluctuation into the objective function; reference [2] studied the scheduling model of electric thermal integrated energy system considering the thermoelectric decoupled cogeneration (CHP) units. However, the common single objective programming is difficult to coordinate multiple scheduling requirements. On the basis of considering the operation cost of electricity to gas in reference [3], the multi-objective weighted fuzzy programming is used to solve the multi-objective scheduling problem in RIES; in reference [4], the entropy weight assignment method of analytic hierarchy process is used to complete the comprehensive evaluation of multiple indexes of power system. However, the accuracy and efficiency of these algorithms are not high. At the same time, how to choose a reasonable optimization solution selection method is also urgent to be solved.

In view of the above problems, taking into account the advantages of demand side response, introducing price type and alternative demand response, a day ahead scheduling plan model is established. With economic optimization as target, the Cplex optimizer is applied to predict the output of each unit of the system. According to the difference of the scheduling time of electric cooling / heating gas and other energy sources, the intra day optimization model including slow control, intermediate control and fast control is established to effectively restrain the occurrence of intra day prediction error and realize the economic and stable operation of the system.

\section{Multi-time scale optimization operation model of integrated energy system}

\subsection{RIES structure diagram}

RIES structure is shown in FIG. 1. The source side includes electric refrigerating machine, cold and hot electricity triple power supply, including gas turbine, waste heat boiler, absorption refrigerator and other cold sources, gas boiler, electric boiler and other heat sources, power grid, wind power, photovoltaic, fuel cell and other power sources, natural gas network, electric gas and other gas sources. Network side includes transmission and distribution, heating/cooling, gas supply and other networks; Storage side includes storage battery, ice storage, heat storage tank, gas storage tank and other equipment for electricity storage, cooling/heat storage and gas storage; The charge side includes cold/hot, electric, gas and other loads.

\footnotetext{
* Corresponding author: szsssxxysh@163.com
} 


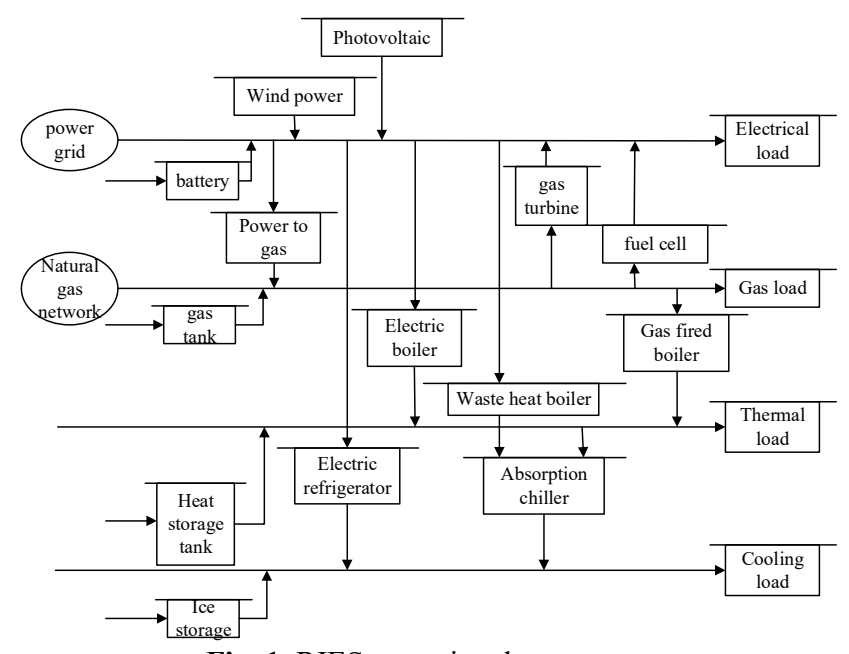

Fig. 1. RIES operational structure.

\subsection{A scheduling}

\subsubsection{The objective function}

Day-ahead scheduling takes scheduling cost minimization as the objective function to make scheduling plan for the next day.

$$
\min F=F_{e}+F_{g}+F_{p}
$$

In the formula, $F_{e} 、 F_{g}$ and $F_{p}$ are the power purchase cost, natural gas purchase cost and equipment maintenance cost in the dispatching period.

$$
\begin{gathered}
F_{e}=\sum_{T=1}^{24}\left(\frac{C_{r s}^{T}+C_{r b}^{T}}{2} P_{\text {grid }}^{T}+\frac{C_{r b}^{T}-C_{r s}^{T}}{2}\left|P_{\text {grid }}^{T}\right|\right) \\
F_{g}=\sum_{T=1}^{24}\left(F_{\text {source }}^{T} C_{\text {gas }}\right) \\
F_{p}=\sum_{T=1}^{24}\left(\sum_{d=1}^{N} C_{d}\left|P_{d}^{T}\right|\right)
\end{gathered}
$$$$
P_{\text {grid }}^{T} 、 C_{r s}^{T} \text { and } C_{r b}^{T} \text { represent the }
$$

In the formula, $P_{g r i d}^{T}, C_{r s}^{T}$ and $C_{r b}^{T}$ represent the power of purchasing electricity, the price of purchasing electricity and selling electricity respectively in the period of T. $F_{\text {source }}^{T}$ represents the natural gas volume purchased in the T period, and $C_{g a s}$ represents the unit price of natural gas. $C_{d}$ is the unit maintenance cost of equipment $\mathrm{D}$, and $P_{d}^{T}$ is the output of equipment $\mathrm{D}$ in the T period [5].

\subsubsection{The constraint}

1) Supply balance constraint

$$
P_{W T}^{T}+P_{P T}^{T}+P_{\text {grid }}^{T}+P_{C C H P}^{T}+P_{E S}^{T}=P_{D m d}^{T}+P_{o}^{T}
$$

In the formula, in the T period, $P_{W T}^{T}$ and $P_{P T}^{T}$ are the electric power generated by WT and PV respectively;
$P_{\text {grid }}^{T}$ is the electric power obtained from the power grid; $P_{C C H P}^{T}$ is the electricity generated by CCHP; $P_{E S}^{T}$ is the output value of ES; $P_{D m d}^{T}$ is the total load power of demand side; $P_{o}^{T}$ is the electric power consumed by coupling equipment.

2) Air supply equilibrium constraint

$$
G_{P t G}^{T}+G_{\text {source }}^{T}+G_{G S}^{T}=G_{D m d}^{T}+G_{o}^{T}
$$

In the formula, $G_{P t G}^{T}$ and $G_{G S}^{T}$ are the natural gas volume obtained from PTG equipment and GS in the T period, $G_{D m d}^{T}$ is the total gas load on the demand side, and $G_{o}^{T}$ is the consumption of coupling equipment.

3) Cooling balance constraint

$$
C_{d}^{T}+C_{C C H P}^{T}+C_{C S}^{T}=C_{D m d}^{T}
$$

In the formula, $C_{d}^{T}, C_{C C H P}^{T}, C_{C S}^{T}$ are $\mathrm{AC}, \mathrm{CCHP}$ and CS output values in t period; $C_{D m d}^{T}$ is total cooling load on demand side.

4) Heating balance constraint

$$
H_{G B}^{T}+H_{E B}^{T}+H_{C C H P}^{T}+H_{H S}^{T}=H_{D m d}^{T}
$$

In the formula, $H_{G B}^{T}, H_{E B}^{T}, H_{C C H P}^{T}$ are the heat generated by GB, EB and CCHP equipment in the T period, $H_{H S}^{T}$ is the HS output of the T period, $H_{D m d}^{T}$ is the total heat load of the demand side in the T period.

\subsection{Intra day rolling optimization scheduling}

\subsubsection{Slow control sublayer scheduling model}

The output of each unit of the system is adjusted according to the change of cooling / heating load in time interval $\mathrm{T}$ according to the operation state and cold / heat dispatching strategy of Mt. The objective function is as follows:

$$
F_{1}=\min \sum_{t=k}^{k+k_{1}}\left(F_{g, 1}+F_{e, 1}\right)
$$

In the formula, $F_{g, 1}$ is the cost of natural gas and $F_{e, 1}$ is the variable cost of electric equipment.

\subsubsection{Middle control sublayer scheduling model}

The output of each unit of the system is adjusted according to the change of natural gas load and slow control layer equipment in time interval $\mathrm{T}$ according to the gas suction and discharge status of GS. The objective function is as follows:

$$
F_{2}=\min \sum_{t=k}^{k+k_{2}}\left(F_{g, 2}+F_{e, 2}\right)
$$


In the formula, $F_{g, 2}$ is the interaction cost with natural gas network, and $F_{e, 2}$ is the variable cost of electric equipment.

\subsubsection{Slow control sublayer scheduling model}

According to the ES charging and discharging state, according to the new energy fluctuation in the $\mathrm{T}$ period and the power changes of the demand side and the two sub levels, the day ahead dispatching is corrected. The objective function is as follows:

$$
F_{3}=\min \sum_{t=k}^{k+k_{3}}\left(F_{e, 3}+F_{\mathrm{g}, 3}+F_{E S}\right)
$$

In the formula, $F_{e, 3}$ is the cost of interaction with power grid, $F_{\mathrm{g}, 3}$ is the cost of natural gas, and $F_{E S}$ is the cost of ES charging and discharging

\section{Demand side response strategy of integrated energy system}

In this paper, the load in the system is divided into traditional load and energy coupling load [6] the traditional load is divided into inevitable load and price load according to the price sensitivity. Energy coupling load can be replaced by other energy to achieve the same energy consumption effect, which is called alternative load. The applicable loads for different demand side responses are shown in Table 1.

\begin{tabular}{cccc}
\multicolumn{3}{c}{ Table 1. Different demand side response loads. } \\
\hline Type & Inevitable type & Price type & Alternative type \\
\hline $\begin{array}{c}\text { Electrical } \\
\text { load }\end{array}$ & $\begin{array}{c}\text { Important loads that must } \\
\text { use electricity }\end{array}$ & $\begin{array}{c}\text { Transferable time } \\
\text { use load }\end{array}$ & \\
Gas load & $\begin{array}{c}\text { Important loads that must } \\
\text { use gas }\end{array}$ & $\begin{array}{c}\text { Transferable time } \\
\text { use load }\end{array}$ & $\begin{array}{c}\text { Electrical, gas } \\
\text { and cooling/ } \\
\text { heating loads in } \\
\text { energy coupling }\end{array}$ \\
$\begin{array}{c}\text { Cooling/ } \\
\text { Thermal } \\
\text { load }\end{array}$ & - & -- & \\
\hline
\end{tabular}

\subsection{Price based demand response}

The above self elasticity coefficient $\eta_{a a}$ represents the response of electricity quantity to the change of electricity price at that time, and the cross elasticity coefficient $\eta_{a b}$ represents the response of electricity quantity to the change of electricity price in other periods.

$$
\begin{gathered}
\eta_{a a}=\frac{\Delta q_{a}}{\Delta p_{a}} \cdot \frac{p_{a}}{q_{a}} \\
\eta_{a b}=\frac{\Delta q_{a}}{\Delta p_{b}} \cdot \frac{p_{b}}{q_{a}}
\end{gathered}
$$

In the formula, $\Delta q$ and $\Delta p$ are the relative increments of electricity quantity $q$ and electricity price $p$ respectively; a and b represent different time periods. Therefore, for periods 1-n, there are the following relations.

$$
\left[\frac{\Delta q_{1}}{q_{1}} \frac{\Delta q_{2}}{q_{2}} \cdots \frac{\Delta q_{n}}{q_{n}}\right]^{T}=E_{e}\left[\frac{\Delta p_{1}}{p_{1}} \frac{\Delta p_{2}}{p_{2}} \cdots \frac{\Delta p_{n}}{p_{n}}\right]^{T}
$$

In the formula,

$$
E_{e}=\left[\begin{array}{cccc}
\eta_{11} & \eta_{12} & \cdots & \eta_{14} \\
\eta_{21} & \eta_{22} & \cdots & \eta_{24} \\
\vdots & \vdots & & \vdots \\
\eta_{n 1} & \eta_{n 2} & \cdots & \eta_{n n}
\end{array}\right] \text { is the }
$$
electricity price elasticity matrix.

Similarly, the natural gas self elasticity coefficient $\omega_{a a}$ and cross elasticity coefficient $\omega_{a b}$ are as follows:

$$
\begin{gathered}
\omega_{a a}=\frac{\Delta g_{a}}{\Delta r_{a}} \cdot \frac{r_{a}}{g_{a}} \\
\omega_{a b}=\frac{\Delta g_{a}}{\Delta r_{b}} \cdot \frac{r_{b}}{g_{a}}
\end{gathered}
$$
matrix.

$$
E_{g}=\left[\begin{array}{cccc}
\omega_{11} & \omega_{12} & \cdots & \omega_{14} \\
\omega_{21} & \omega_{22} & \cdots & \omega_{24} \\
\vdots & \vdots & & \vdots \\
\omega_{n 1} & \omega_{n 2} & \cdots & \omega_{n n}
\end{array}\right] \text { is the gas volume and price }
$$

\subsection{Alternative demand response}

In IES, electricity, natural gas and cold / hot energy are converted between different energy sources through relevant coupling equipment. The energy conversion follows the law of conservation of energy, and the conversion relationship of alternative demand response is as follows.

$$
\begin{gathered}
\Delta R_{i}=-\gamma_{i, j} \Delta R_{j} \\
\gamma_{i, j}=\frac{W_{i} \cdot \eta_{i}}{W_{j} \cdot \eta_{j}} \\
\Delta R_{i}^{\min } \leq \Delta R_{i} \leq \Delta R_{i}^{\max } \\
\Delta R_{j}^{\min } \leq \Delta R_{j} \leq \Delta R_{j}^{\max }
\end{gathered}
$$

In the formula, $\mathrm{i}$ and $\mathrm{j}$ represent two kinds of energy, such as electricity, natural gas, cold and heat respectively; $\Delta R_{i}$ and $\Delta R_{j}$ are the load increment of two kinds of energy, $\gamma_{i, j}$ is the conversion coefficient between energy; $W_{i}$ and $W_{j}$ are the unit calorific value of energy $\mathrm{i}$ and $\mathrm{j}$ respectively; $\eta_{i}$ and $\eta_{j}$ are the utilization rate of energy $\mathrm{i}$ and $\mathrm{j}$ respectively; $\Delta R_{i}^{\min }, \Delta R_{i}^{\max }$ and $\Delta R_{j}^{\min }, \Delta R_{j}^{\max }$ are the minimum and maximum load increment of energy $i$ and $j$. 


\section{3. demand side response strategy under multi time scales}

Before dispatching, the electrical load adjusted by the unavoidable load and the price type in the traditional class is preliminarily fitted, and the cold / heat load value required by the coupling device is determined. In the intra day rolling optimization, according to the day ahead pre scheduling plan, the influence of demand side fluctuation is further considered, and the alternative response is used to optimize the energy layer by layer.

\section{Case analysis}

\subsection{Example parameter setting}

In this paper, a regional ies is selected for analysis, and the system structure is shown in Figure 1. The self elasticity coefficient of electricity price is -0.2 , the cross elasticity coefficient is 0.03 [7]; the self elasticity coefficient of price type natural gas is -0.58 , and the cross elasticity coefficient is 0.15 [8].

\subsection{Daily scheduling analysis}

The system can meet the balance of supply and demand when the electric cooling and heating gas networks are coupled. During the period from 23:00 to 07:00 of the next day, when the electricity price is in the low valley, the system will guide the electric energy through EB, convert the electric energy into heat energy and give priority to the output; through the PTG device, the electric energy will be converted into natural gas to increase the output of PTG; the guiding electric energy will be converted into cold energy through $\mathrm{AC}$ equipment to meet the cold load. When the electricity price is in the peak period, MT will increase the output to replace the interactive power with the grid by generating electricity, and increase the discharge of ES to meet the demand of electric energy, such as 18:00 to 20:00; it will also guide PTG to reduce its output, such as 10:00 to $14: 00$; the cooling load mainly depends on $\mathrm{AR}$, such as 10:00 to $15: 00$; the heat load mainly depends on WHB to meet the demand, such as 18:00 By 21:00. The time of use price of electric energy and natural gas is different in peak and trough periods, which further reduces the cost.

\subsection{Intra day rolling optimization scheduling analysis}

The results of day ahead and day-to-day optimal scheduling show that the output of coupling equipment and the interactive power of electrical and external network are corrected in the day. The interactive power curve with the power grid fluctuates with the change of electricity price, and the amount of electricity purchased during the peak period is significantly reduced to achieve economic optimality. When the price of natural gas is high, MT is driven to keep the balance of supply and demand of the system. When the price of natural gas is high, the output of MT is reduced. The daily output of the equipment will be more accurately scheduled for cold energy.

\section{Conclusions}

In this paper, based on the characteristics of demand side response of price type and alternative type, considering the difference of dispatch time of electricity, cold / heat and gas, a multi time scale optimal scheduling model of ies considering demand side response is proposed.

(1) The demand side response is related to the scheduling efficiency of IES. When the system is scheduled according to the model proposed in this paper, the economic operation cost is optimal, which can better reflect the advantages of demand side response.

(2) Based on the results of day ahead optimization, the time scale is further shortened and the accuracy of system decision is improved.

(3) The daily three-level rolling optimization scheduling model can dispatch electricity, cold / hot and gas in different time scales, so that the system can suppress the fluctuation of both supply and demand, and ensure the stable operation of the system.

\section{References}

1. WEI Zhinong, ZHANG Side, SUN Guoqiang, et al . Power-to-gas considered peak load shifting research for integrated electricity and natural-gas energy systems $[\mathrm{J}]$. Proceedings of the CSEE, 2017, 37( 16) : 4601-4609, 4885 .

2. WEI Zhenbo, HUANG Yuhan, GAO Hongjun, etal . Joint economic scheduling of power-to-gas and thermoelectric decoupling CHP in regional energy Internet [ J ] . Power System Technology, 2018, 42( 11) : 3512-3520.

3. DONG Shuai, WANG Chengfu, LIANG Jun, et al . Multi-objective optimal day-ahead dispatch of integrated energy system considering power-to-gas operation cost $[\mathrm{J}]$. Automation of Electric Power Systems, 2018, 42(11): 8-15, 121.

4. DONG Fugui, ZHANG Ye, SHANG Meimei . Multi-criteria comprehensive evaluation of distributed energy system $[\mathrm{J}]$. Proceedings of the CSEE, 2016, 36( 12):3214-3223.

5. Correa-Posada C M, Sanchez-martin P, Lumbreras S. Security-constrained model for integrated power and natural-gas system[J]. Journal of Modern Power Systems and Clean Energy, 2017, 5(3): 326-336.

6. Zhang Yining, He Yubin, Yan Mingyu, et al. Optimal dispatch of integrated electricity-natural gas system considering demand response and dynamic natural gas flow $[\mathrm{J}]$. Automation of Electric Power Systems, 2018, 42(20): 1-10. 
7. Xue Yuntao, Chen Yichao, Li Xiuwen,et al Ramsey Federation model of TOU and ladder price based on customer satisfaction and Ramsey pricing [J]. Power System Protection andControl, 2018, 46(5): 122-128.

8. Li Lanlan, Zhu Kejun, Yang Juan. Literature review on natural gas demand price elasticity [J]. Journal of Beijing Institute of Technology(Social Sciences Edition), 2012, 14(6): 22-31. 